Document downloaded from:

http://hdl.handle.net/10251/63784

This paper must be cited as:

Acedo Rodríguez, L.; Lamprianidou, E.; Moraño Fernández, JA.; Villanueva Oller, FJ.; Villanueva Micó, RJ. (2015). Firing patterns in a random network cellular automata model of the brain. Physica A: Statistical Mechanics and its Applications. 435:111-119. doi:10.1016/j.physa.2015.05.017.

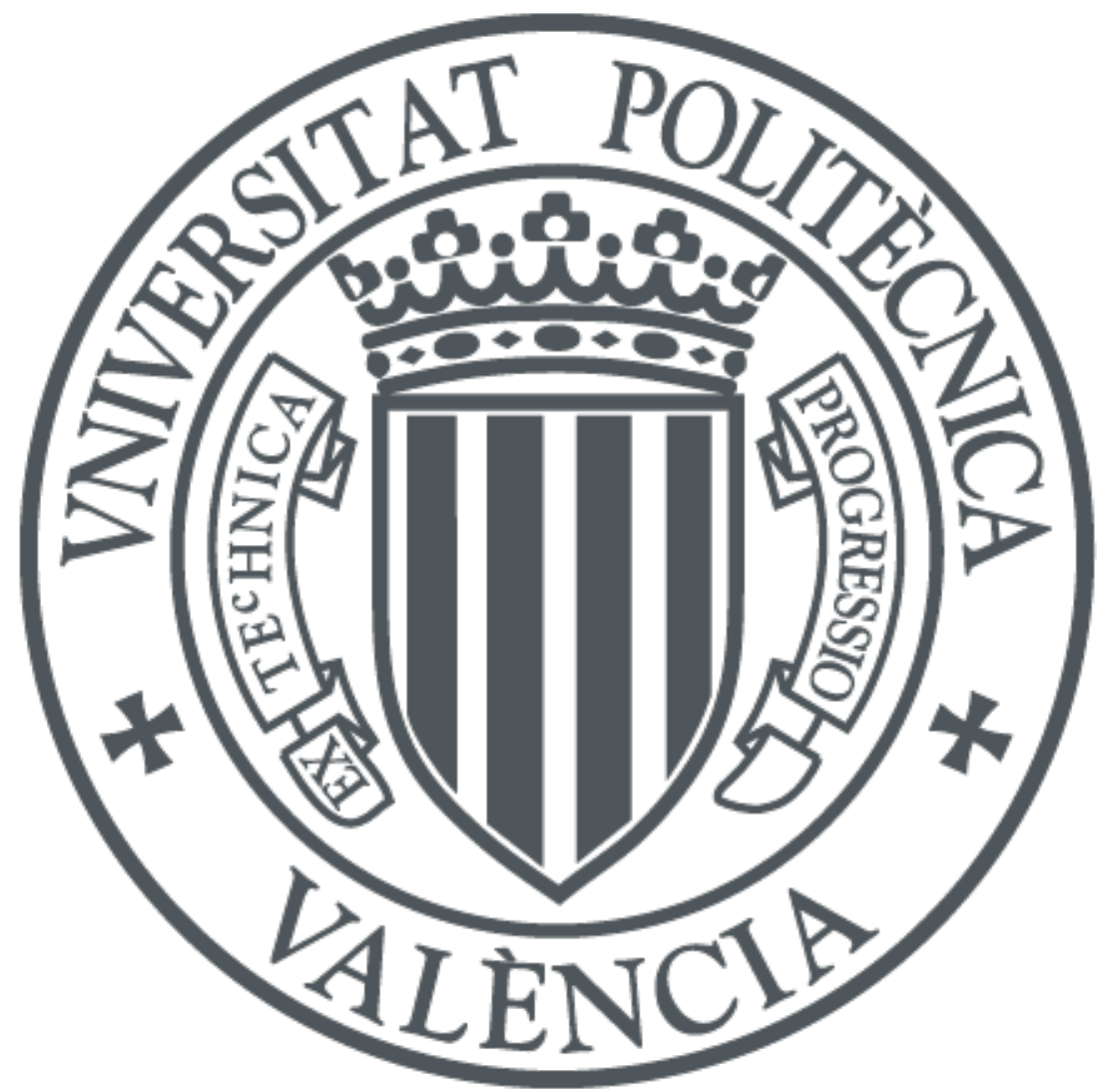

The final publication is available at

http://dx.doi.org/10.1016/j.physa.2015.05.017

Copyright Elsevier

Additional Information 


\title{
Firing patterns in a random network cellular automata model of the brain
}

\author{
L. Acedo ${ }^{b}$, E. Lamprianidou ${ }^{b}$, J.-A. Moraño ${ }^{b}$, \\ J. Villanueva-Oller ${ }^{\star}$ and R.-J. Villanueva ${ }^{b}$
}

(b) Instituto Universitario de Matemática Multidisciplinar, Building 8G, Door C, Second Floor, Universitat Politècnica de València, 46022 Valencia, Spain

( $\star$ ) Centro de Estudios Superiores Felipe II, Aranjuez, Madrid, Spain

\begin{abstract}
One of the main challenges in the simulation of even reduced areas of the brain is the presence of a large number of neurons and a large number of connections among them. Even from a theoretical point of view, the behaviour of dynamical models of complex networks with high connectivity is unknown, precisely because the cost of computation is still unaffordable and it will likely be in the near future. In this paper we discuss the simulation of a cellular automata network model of the brain including up to one million sites with a maximum average of three hundred connections per neuron. This level of connectivity was achieved thanks to a distributed computing environment based on the BOINC (Berkeley Open Infrastructure for Network Computing) platform. Moreover, in this work we consider the interplay among excitatory neurons (which induce the excitation of their neighbours) and inhibitory neurons (which prevent resting neurons from firing and induce firing neurons to pass to the refractory state). Our objective is to classify the normal (noisy but asymptotically constant patterns) and the abnormal (high oscillations with spindle-like behaviour) patterns of activity in the model brain and their stability and parameter
\end{abstract}

*e-mail: luiacrod@imm.upv.es 
ranges in order to determine the role of excitatory and inhibitory compensatory effects in healthy and diseased individuals.

Keywords: Networks dynamics, Cellular automata, EEG, Random networks.

\section{Introduction}

At the turn of the nineteenth century, Santiago Ramón y Cajal observed the detailed microscopic structure of the nervous system thanks to the staining method developed by Golgi. These breakthroughs settled the foundations of modern neuroscience after the proposal of the so-called neuron doctrine by von Waldeyer-Hartz in 1891, that is, the fundamental notion that the nervous system is composed of individual cells. Later on, Ramón y Cajal proved the directionality of synaptic contacts among neurons and showed that these contacts are contiguous but not continuous as Golgi defended [1]. These landmarks set the beginning of modern neuroscience and, in particular, since Ramón y Cajal the brain is considered a very intricate network, although it was not called this way at those early times [2]. Ramón y Cajal was the first researcher to explore the anatomy of the brain tissue on a cellular level in full detail. The human brain, in particular, is composed by a set of $10^{10}-10^{11}$ individual neurons but, as impressive as this large number seems, the total number of connections is even larger because every cell projects its synapses to a total of $10^{4}-10^{5}$ different neurons [3]. So, the statement that the brain is a network is solidly supported by physiological evidence but the practical implementation of the concept in mathematical and physical models has remained in shadows until the emergent science of networks was developed.

Cellular automata (CA) is another important concept for complex systems [4]. In these models every site has different, usually Boolean, states and it evolves according to a rule or set of rules which indicates how the state of a single automaton changes according to the state of its neighbours. It was shown that these simple models and rules could produce interesting complex behaviour as those found in real complex systems [5]. Consequently, it seems natural to use both concepts, networks as well as cellular automata, as the most economic way to build a model of the brain.

Another important feature of the brain tissue is the presence of two distinct types of neurons: excitatory and inhibitory ones $[6,7]$. In the excitatory 
neurons the action potential which propagates through their dendrites gives rise to the liberation of the glutamate neurotransmitter at the synaptic cleft. Glutamate is the main excitatory neurotransmitter in the brain and, if the action potential in the presynaptic neuron is sufficiently intense, it could induce excitation on the postsynaptic neuron. If the postsynaptic neuron is finally excited it could react back upon the initial excitatory neuron (but as it is inhibitory it would deactivate the initial neuron or modulate its firing). Inhibition in this case is mediated by the $\gamma$-aminobutyric or GABA, which is the main chemical causing inhibition in the brain. It is known that inhibitory neurons constitute up to a third of the total neural population in any brain tissue and their role as modulators of the global brain activity is key in the prevention of aberrant firing patterns. In particular, neural network dysfunctions in the excitatory and inhibitory circuits have been proposed as a mechanism in Alzheimer's disease [8]. A failure in the inhibitory GABAergic circuits is also related to a wide variety of conditions ranging from epilepsy [9] to Huntington's disease [10].

The control of rhythmic activity in organisms with a central nervous system has also been associated with the synchronization of the patterns of activity of many neurons in the mesoscopic and macroscopic scales [11]. However, the details of the synchronization mechanisms are still under debate and many mathematical models have been proposed: Jiao and Wang studied recently of phase coupling functions in a model of a neuronal population with excitatory and inhibitory connections [12]. Feedback control and delays in the coupled connected neural networks have also been considered [13, 14, 15]. Kavasseri and Nagarajan also studied the synchronization of electrically coupled neural networks [17] using Izhikevich model for individual neurons [16] and analyzing the role of conductance of the junctions as well as the density of connections. Synchronized bursting of a real in vitro network was observed by Segev et al. [18] who also studied the dependence of $C a$ concentration. But the emergence of oscillatory patterns could happen at the mesoscopic level of the network without the need of a complex dynamics of the neurons. To investigate to what extent the complexity of the microscopic structure is necessary to explain collective behaviour of the brain, cellular automata models can be used. Determining the universality class in which the brain as a complex system can be classified [19] is also a fundamental issue with important consequences for the engineering and biology of neural networks.

In this paper we propose a basic mathematical model implementing the 
fundamental structural and dynamical properties of the brain, that is, its high connectivity per neuron and the presence of two populations (excitatory and inhibitory) with competing effects. With this model we will be able to determine the range of parameters in which high collective oscillations and normal stable behaviour can be found. The emergence of abnormal patterns of oscillations with high amplitude is found for a restricted set of parameters. These behaviour could be related to epilepsy and other medical conditions. The insight obtained with the CA model may favour the scenario of a deficit in GABAergic inhibition as the source of most cases of epilepsy [20] and emphasize its origin in the mesoscopic level rather than the microscopic details of the neurons.

This paper is organized as follows: In Sec. 2 we propose the cellular automata network model and discuss its implementation in a distributed computing environment. Data retrieval and analysis are presented in Sec. 3. In this section we also discuss the phase diagram obtained by classifying the behaviour into oscillatory or constant signal with random statistical noise. Finally, we end the paper with some remarks and a plan for future work in Sec. 4.

\section{A cellular automata network model of the brain}

The most natural sparse generalization of the complete graph in mathematics is provided by the so-called Erdös-Rényi graphs [21], which in the fifties of the past century defined the concept of a random graph. In modern times, this idea has become redefined as a random network and it has become an important paradigm with many applications. Other alternatives are the small-world model of Watts and Strogatz [22] or the scale-free network [23].

The cytoarchitecture of the human cortex is characterized, in general, by stratified layers of neurons. This basic structure is already present at birth but dendritic arbors develop and grow during the first two years [24]. It is known that the details of this fine substructure develop throughout many years. In our approach, we are more interested in the topological properties of these networks and we should ignore the stratified architecture. We choose the Erdös-Rényi random network model characterized by a Poisson distribution of contacts among nodes with a mean value $k$ as a good approximation 
to the average connectivity properties in the real brain. Random networks are characterized by the number of sites or nodes $\left(N=10^{6}\right.$ in our simulations) and the average number of contacts of every individual, $k$ (called the degree of the node, in our simulations we consider $k=300$ ). Consequently, the number of links in the network is given by $N k / 2$. These links are randomly assigned to pairs of nodes with the obvious rule that, at most, only a link can connect two nodes. By following this algorithm we find a Poisson distribution for the degree of the nodes [25].

The values of $N$ and $k$ used in this study are relatively low compared with those of the human brain. Particularly, $k$ is still small in comparison with the average $k=10000$, typically found in humans. This limit has been set by the limited resources of the computers of the volunteers involved in the BOINC project to be discussed below [26]. The most sophisticated reverse-engineering of the human brain, the so-called Human Brain Project [27], involves values of $k$ in the range of 1000 to 10000. Nevertheless, there are problems in which a reduced number of neurons and connections could prove useful and, moreover, the results are easily reproducible by research teams without the computational resources to carry out a study on the larger scale. The BOINC procedure we will discuss in this section provides such a computational tool.

On the other hand, the neurobiology of mini-brains, such as those of social insects, is a very active area of neuroscience research nowadays. In the case of the honey bee the average number of neurons has been estimated to be 960,000 neurons with an average of 1000 synapses per neuron [28]. Drosophila's brain has only $k=10$ synapses per neuron [29] but these insects have a very complex behavioural repertoire. Developing neural network models for these small brains is, at present, a challenge more manageable than attacking the problem of the human brain but, still, it is highly interesting from the point of view of the evolutionary biology of the nervous system [30]. In particular, a question that should be addressed concerns the minimum size and connectivity of a neural network capable of exhibiting synchronization and oscillatory patterns of activity. The role of synchronous firing in insects has been found to be connected with odour discrimination [31] and these appears to be the smallest nervous systems where a functionality for these neural behaviour is disclosed.

It is an empirical fact that, approximately, a thirty per cent of the cortical neurons are inhibitory [35]. So, in any minimally realistic model of the brain we must consider both excitatory and inhibitory neurons. The excitatory 
ones can induce the firing of their postsynaptic neighbours by the pulses they send when they are firing. On the other hand, the inhibitory neurons produce the opposite effect, i. e., they prevent other neurons from firing or turn firing neurons into the refractory state.

According to its temporal state, neurons are classified into three states: resting, firing and refractory. Resting neurons can be stimulated by the synaptic inputs from presynaptic firing neurons. On the other hand, firing neurons send electrochemical spikes along their synapses towards other neurons. After the firing phase, neurons become refractory and cannot be stimulated to the firing state again until they turn into rest. The refractory state lasts for several milliseconds. In this state, the potential of the membrane is below the resting potential and this prevents the refractory neuron from activating.

Once the random network is generated we apply an evolution algorithm in order to analyse the number of active neurons as a function of time. As initial state we consider only a small fraction of firing neurons. All quiescent neurons are then checked iteratively and they start to fire at time step $t+1$ with probability $\alpha$ for every contact with an excitatory firing neuron at time $t$. The contacts with firing inhibitory neurons may prevent the firing with a certain probability, $\alpha$, or deactivate a firing neuron with the same probability.

The average time a neuron remains in the firing state is $1 / \nu$ whereas the average refractory time is $1 / \lambda$. These firing and refractory times are intrinsic to the neuron structure and we assume that they follow a Poisson distribution with the aforementioned averages. In our stochastic model we are simulating the transmission of action potentials from neuron to neuron as a stochastic process. In practice, neurons integrate the signals received from the axon projections of their neighbours. This is the basic idea of the socalled integrate-and-fire. At every instant $t$, and for every quiescent neuron, we analyse one by one the connections that it has with firing neurons. If one connection is to an excitatory firing neuron, our neuron becomes a firing neuron with a probability of excitation $\alpha$, or it stays as it is and we move on to analyse the next connection. If the analysis shows that the quiescent neuron is activated and becomes a firing neuron, at the next connection it is examined if the contact is with an inhibitory neuron. If it is in fact, our firing neuron is deactivated with an inhibition probability $\gamma$ and becomes a quiescent neuron. Following this process all the neuron connections are analysed until the neuron finally remains as quiescent or firing, before changing to another neuron at the same instant $t$. 
Modelling neurons as cellular automata is really a very simple assumption because individual neurons exhibit themselves many complex firing patterns [7]. Moreover, inhibitory and excitatory neurons are quite different in their behaviour and models based upon differential equations capture the essential difference [16]. However, we are interested in finding the minimum complexity necessary in a network model to allow for the emergence of synchronous and hypersynchronous oscillations in the number of firing neurons. We will show that oscillatory patterns emerge at the mesoscopic level without implying complex firing in the microscopic level. Hogdking-Huxley [32], FitzHugh-Nagumo [33] or the simpler Izhikevich's neurons [16] could replace the cellular automaton in our model and this surely will change the parameter ranges where oscillatory behaviour is found but the CA model will reveal the possible mesoscopic nature of oscilations arising from the competing effects of excitation and inhibition in a random network.

Notice also that the time-step in the CA model is arbitrary but, in case of looking for a correspondence with the real brain, we should take it as a fraction of a millisecond to allow for the sufficient temporal resolution. The time-scale is really fixed by the values of the transition probabilities from the firing to the refractory state, $\nu$, and from the refractory state to quiescent, $\lambda$. As the duration of the action potential is in the range 1-2 milliseconds, we could take $1 / \nu=1.5$ milliseconds and $1 / \lambda=2$ milliseconds because the refractory period is in the range 1-3 milliseconds [7]. Spike trains are usually modelled as events of a slowly varying inhomogeneous Poisson process [34] (this is sometimes known as the Poisson hypothesis) and the use of Poisson processes to model the duration of the firing and refractory states in the cellular automata network could account for this variability in the arrival of the spikes.

It is difficult to ascribe a definite value to $\alpha$ or $\gamma$ according to our present understanding of brain physiology. The measurements of cerebral glucose utilisation by means of the positron emission tomography technique has revealed a decline in several areas of the brain during sleep [36]. Vyazovskiy et al. [37] also found that periods of neural silence are long and frequent during early non-REM sleep after prolonged wakefulness. On the other hand, Grosmark et al. have also recently found that the alternating REM and non-REM episodes during sleep are characterized by different hippocampal excitability [38], implying a possible neuromodulation of firing in the sleep states. These observations are consistent with a scenario in which the homeostatic equilibrium of neurotransmitters is different between sleep states and the awaken 
state and could be mimicked by adjusting the values of these probabilities. Sleep homeostasis also manifests itself in an increase of the need of sleep after long periods of wakefulness. In a previous work, one of us showed that, under the assumption that EEG is a statistical epiphenomenon at the mesoscopic level, we can qualitative match the high amplitude deep sleep signals and the low amplitude alpha rhythms with different phases of a CA model in a complete graph [39]. The monitoring of neuronal avalanches from intracranial depth recordings in slow wave sleep, wakefulness and REM states also shows different behaviour concordant with small changes in synaptic strength [40]. In principle, the neuronal avalanche distributions could also be simulated by the CA model in order to obtain a measure of the synaptic strength in each state in terms of the excitation and inhibition probabilities. Moreover, in the case of external stimuli that promote the opening of sodium channels in sensory neurons the role of $\alpha$ or $\gamma$ is clearer as a probability that measures the increase or decrease of firing probability of the stimulated neurons. Furthermore, the mean firing time, $1 / \nu$, and the mean refractory time, $1 / \lambda$, can also be obtained from the physiology of a single neuron. However, in this work we are concerned about the general behaviour of the simulated brain networks and we will not attempt to give a definite correspondence with the brain physiology.

In the CA model the strength of the synapses is represented by the excitation, $\alpha$, or inhibition probabilities, $\gamma$, considered to be constants throughout the whole network. However, we could also consider the case of inhomogeneous synapses with probabilities varying from synapse to synapse. These probabilities could also evolve according to Hebbian learning rules [41]. But the role of plasticity and varying weights between neurons is not to be discussed in this paper because our primary objective is to identify the characteristic behaviour of the model with constant synapses. Evolving networks would also require more computational power and, even with distributed computing techniques, will surpass reasonable computation times in a typical mainframe.

Summarizing, the algorithm for the simulation of the Cellular Automata Random Network model should include the following steps:

- A random network for $N$ sites and average degree $k$ is generated by randomly assigning $N k / 2$ bonds to different pair of sites. A pair of sites is chosen randomly among all pairs of sites without bonds connecting them and a bond is assigned until the total number $N k / 2$ is exhausted. 
The network is different on every realization although their statistical properties are the same.

- A fraction (usually a $30 \%$ ) of sites is labelled as an inhibitory neuron. The rest are labelled as excitatory.

- The network is initialized by choosing a fraction of neurons in the firing state (the rest being at the resting state).

- For every resting neuron in the network we check its neighbours and, if they are excitatory firing neuron, the resting neuron changes its state to firing with probability $\alpha$. Similarly, if a neighbour is an inhibitory firing neuron the state of the test neuron changes again to resting with probability $\gamma$ if it has been stimulated by an excitatory firing neuron in its neighbourhood during this step.

- Every firing neuron can become a refractory neuron with probability $\nu$ per step. Similarly, the firing neuron becomes refractory with probability $\gamma$ for every inhibitory firing neuron in its neighbourhood.

- Refractory neurons change to the resting state with probability $\lambda$ per time step.

- At every time step we count the number of excitatory and inhibitory firing neurons as a measure of the network's activity.

\subsection{Distributed Computing}

Simulations for cellular automata network models is a very demanding task for the computers available today, especially for very large number of sites $\left(10^{6}\right)$ and high connectivity degrees. As we intend to carry out simulations in networks with one million sites and an average of 300 connections per site, it was clear that a distributed computing solution is necessary if we plan to obtain results for many parameter combinations in a reasonable period of time.

Consequently, we have tried out BOINC (acronym for Berkeley Open Infrastructure for Network Computing). BOINC is an open source software which has become the standard in many collaborative projects all around the world [26]. Among the most renowned projects are SETI@home, ROSETA or Climate Prediction. BOINC protects against several types of attacks and 
the distribution of viruses by using digital signatures based on public-key encryption, its server architecture is highly scalable and the core client is available for most common platforms.

The name of our projec is Neurona@Home and it is coordinated from the CES Felipe II in Aranjuez, Madrid, Spain which provides ad-hoc BOINC deployment and computing power for small to medium computation problems [42]. The solver was adapted to the requirements of the problem and the project was launched to the Internet community in 2011. The implementation of BOINC projects for epidemiological models has been discussed in full detail in a previous publication [43]. Some preliminary results for the Neurona@Home project were already published [44].

With the increased computation power of the many volunteers of the BOINC community we were able to simulate more than 155000 tasks in a reasonable time. We must take into account that the computers necessary to perform the simulations require both a fast $\mathrm{CPU}$ and, at leat, 8 Gygabytes of RAM to store a network with $k=300$. With this large number of tasks processed, a reliable picture of the phase diagram can be obtained as shown in the next section.

\section{Simulation of the model and data analysis}

As mentioned in the previous section our objective has been to simulate a large-scale $\left(N=10^{6}\right.$ nodes) network model with the evolution rules for the individual cellular automata mimicking those of excitatory and inhibitory neurons. After several months of computer time in the Neurona@Home project we obtained 155,169 tests with 1,500 time intervals. The tests correspond to different combinations of the parameters: $k$, average degree of the random network, $\alpha$, excitation probability, $\gamma$, inhibition probability, $1 \nu$, average time that a neuron (without other interactions with their neighbours) passes in the firing state and $1 / \lambda$, average time that a neuron spends in the refractory state before becoming a resting neuron again.

The parameter space has five dimensions and it is really beyond the capabilities of modern computers and distributed computing environments to study in full detail. Consequently, we restricted our study to networks with $k=300$ (an average connectivity much larger than those studied before but still small in comparison with that of the human brain, i.e., $k=10000$ ). Moreover, we assume that the excitation probability and the inhibition prob- 
ability are identical, $\alpha=\gamma$.

The parameter range we have explored is the following:

$$
\begin{aligned}
k & =300 \\
1 / \nu & =1,2, \ldots, 10 \\
1 / \lambda & =1,2, \ldots, 100 \\
\alpha=\gamma & =0,0.001,0.002, \ldots, 0.0009 .
\end{aligned}
$$

Notice that for the realization of the tests, the values of the probabilities $\alpha$ and $\gamma$ have been considered the same, even though they could have been selected to be different. Moreover we studied some cases where $\alpha$ and $\gamma$ were different but we did not obtain oscillatory behaviour. It is known that the strength of inhibitory connections is usually higher than those of excitatory ones and, moreover, inhibitory terminals suffer less depression than excitatory terminals after sustainted activation [45]. As we are particularly interested in the emergence of hypersynchronous oscillations associated to epileptic seizures a reduced inhibition is reasonable to be considered because reduced GABAergic inhibition is expected in epileptic patients [20].

In a previous study we also considered the emergence of oscillatory behaviour as the number of neurons in the CA network is increased up to $10^{6}$ [43]. We found that, at least, half a million neurons are needed to assure the stability of these oscillations in the network. Interestingly, this number of neurons marks the evolutionary leap represented by the mini-brains of insects which use these oscillations as an odour-encoding algorithm [31].

The tests were performed at the Polytechnic University of Valencia and also at the CES Felipe II of Aranjuez. The time required to perform all the tests was approximately 44 weeks.

Since we have in our possession a large number of tests, we need to discriminate the ones that have led to oscillatory behaviour. We are searching for patterns of organized activity similar to that of an electroencephalogram. To accomplish this task, two types of discrimination are used in our investigation. For this discrimination we have utilized inhibitory neurons that are excited. Firstly, the discrimination with the coefficient of variation, $\mathcal{C}_{v}$, is utilized. The coefficient of variation indicates a statistical measure of the distribution of data points in a data series around the mean. It states the ratio of the standard deviation, $\sigma$, to the mean, $\mu$. Furthermore, we will apply the Discrete Cosine Transform or DCT-based oscillation detection method [46] to proceed with the discrimination task. The DCT is utilized in our research 
since it only involves the use of cosine functions and real coefficients.

The coefficient of variation $\left(\mathcal{C}_{v}\right)$, is a large number, provided that $\mu$ is not a very small number. Due to this fact, we have considered as candidates all the tests that exceed the number 0.5 when calculating the ratio $\sigma / \mu$ of the last 750 values. If it is in fact larger, we save this test, otherwise it is disregarded. In combination with this, we specify that the mean value of 200 last values is larger than 100 .

The first values are not considered in the calculations of $\mu$ and $\sigma / \mu$, since the signal is expected to become stabilized from the initial conditions. This way signals with mean values very close to zero are dismissed, even though the values of $\sigma / \mu$ would result very large. This is due to the fact that the signals obtained from the brain would not be interesting for investigation. By the end of this procedure, we acquire the tests that fulfill these traits:

$$
\begin{aligned}
C & =\frac{\sigma}{\mu} \geq 0.5 \quad \text { (for the last } 750 \text { values) } \\
\text { while } \mu & >100 \quad \text { (for the last } 200 \text { values). }
\end{aligned}
$$

These threshold values were chosen by trial and error in such a way that they provide an optimum classification of the modelled signals. A check for periodicity was performed in the next stage and complemented the initial screening. After this simple screening, the total number of candidate tests is reduced to 3436 .

In the following we shall study the second discriminating method. By representing the first coefficient of their DCTs found in each of the 3436 tests we get Fig. 1. From Fig. 1, we decided to use 100000 as a point of reference in order to separate the graph in two blocks. The upper block involves 3356 tests and the block below 100000, contains 80 tests. All tests whose first coefficient of its DCT is bigger than 100000, we speculate that can be of oscillatory and periodic behaviour. On the contrary we consider that the points of the lower block have a noisy behaviour. The tests with the desired maximum DCTs are the ones in the upper block.

After implementing the first discrimination process, we represent graphically the 500 last values for each one of the 3436 candidate tests and at the same time their discrete cosine transform. We obtained a list of figures, two characteristic examples of which are shown in Figs. 2 and 3.

We observe that for some tests we get a non-periodic behaviour and very small values of the DCT (Fig. 2). On the other hand, from other tests we obtain graphs with periodic, oscillatory behaviour with a much larger DCT 


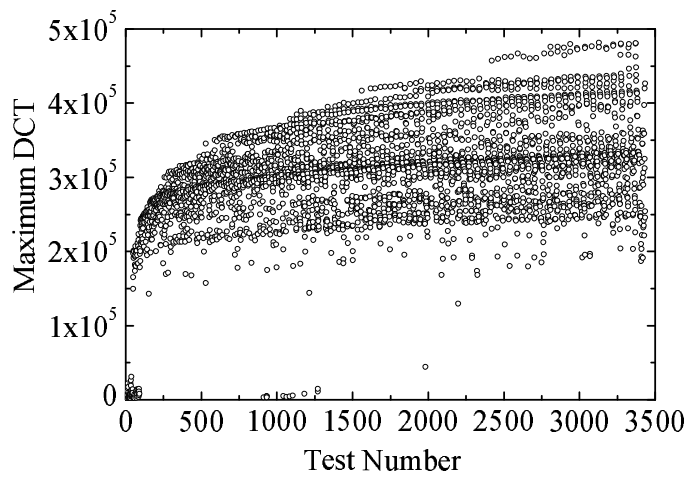

Figure 1: Representation of the first coefficient of its DCTs. The tests obtained by the filter of the $\mathcal{C}_{v}$ are represented according to the maximum value of the DCT. We can see that the majority of the values are in the interval $\left(10^{5}, 5 \times 10^{5}\right)$, but still there are some tests below this strip.
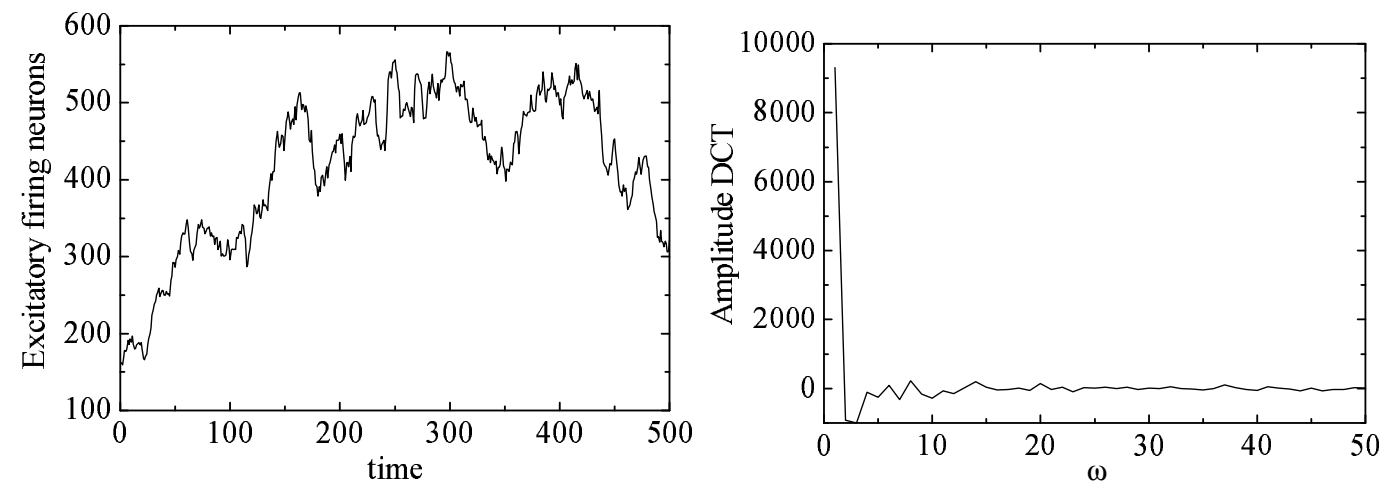

Figure 2: Number of firing excitatory neurons in a typical test with nonperiodic behaviour (left) and the corresponding DCT (right). 

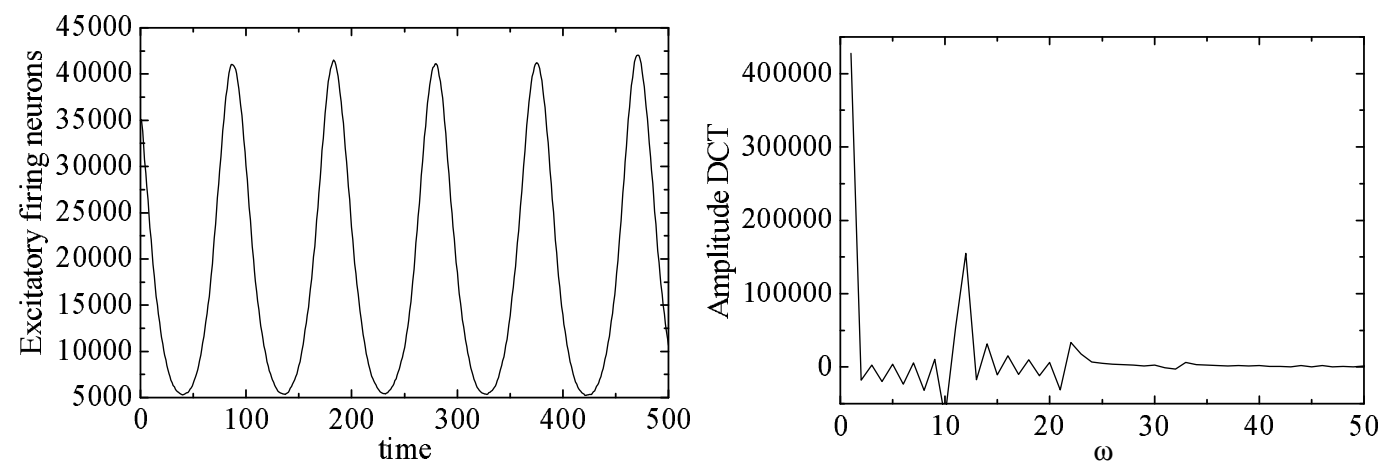

Figure 3: The same as 2 but for a test with periodic behaviour.

value (Fig. 3). These last mentioned behaviours appear in a greater scale than the non-periodic ones. Furthermore, the rank where the DCT of the periodic ones is moving is greater than that of the non-periodic ones. In particular the rank of the non-periodic ones fluctuates between -10000 and 10000 while the rank of the periodic ones is between -100000 and 500000 . Further on, by using the second discrimination method of the first coefficient of their DCTs on the 3436 candidate tests we found that 3356 tests have a periodic behaviour while 80 tests present a non-periodic one.

\subsection{The phase diagram}

We have explored the region in which the regular oscillatory behaviour in Fig. 3 is obtained. The regions of periodic and non-periodic behaviour are displayed in Fig. 4 in terms of the three free parameters $1 / \nu, 1 / \lambda$ and $\alpha=\gamma$ for a network with $10^{6}$ neurons and an average degree $k=300$. The regular oscillatory behaviour is only observed in a very restricted region of the three-dimensional parameter space. Consequently, for most combinations of parameters the number of firing neurons is an almost constant but noisy time series. This is in correspondence with the behaviour of the real brain: it is a commonplace in neuroscience that electroencephalograms (EEG) are noisy signals with a spectrum $1 / f$ which has been associated with self-organized criticality [47] although, in the case of the brain, that explanation has been dismissed [48]. On the other hand, in some clinical cases, a regular synchronous oscillation appears. In the case of epilepsy this happens in the so-called ictal state characterized by the paroxysmal ocurrence of large amplitude oscillations in the EEG. As this occurs only in very particular situ- 
ations, even for epileptic patients, we can say that the model in this paper captures the rarity of these events because most of the activity is interictal, i. e., a normal noisy state of low amplitude.

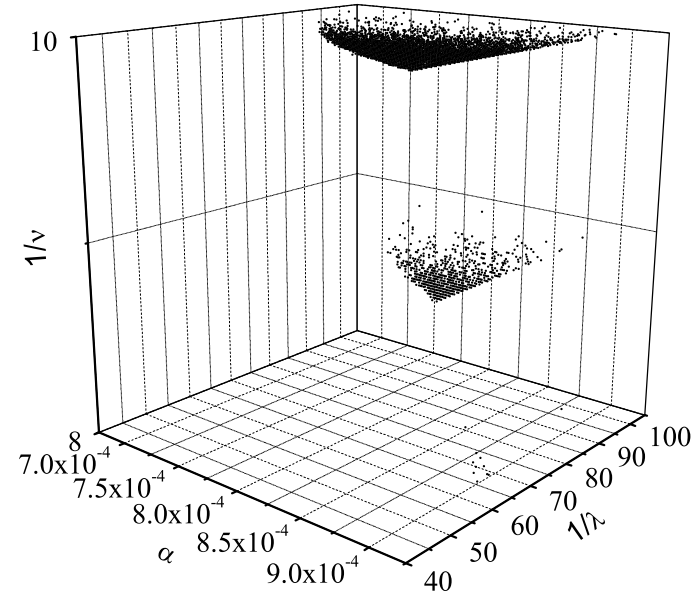

Figure 4: Three-dimensional phase diagram for the ocurrence of oscillatory behaviour in the number of firing neurons in the network. The parameters in the axes are: $1 / \nu$, average transition time from firing to refractory, $1 / \lambda$, average transition time from refractory to resting, and $\alpha$, probability of stimulation of a resting neuron by a firing excitatory neuron which, in our simulations, is assumed to be equal to $\gamma$, the probability for a firing inhibitory neuron to deactivate a neighbouring firing neuron.

\section{Discussion and conclusions}

Cellular automata are an important paradigm in statistical mechanics with a broad range of applications to complex systems. Comparatively, there have been few implementations of this concept in the context of modelization of the brain, as the primordial example of a very complex system $[49,50]$. On the other hand, networks have evolved into a field of research in itself which have also received considerable interest in the last three decades. Combining these ideas we have defined a network of cellular automata as an elementary model of the brain implemented as a network of neurons.

In this work we have simulated this stochastic neural network model of $10^{6}$ neurons with a distributed computing system that has allowed us to carry out 
about 155000 tests in a reasonable time. The objective was to find collective behaviour of the network that can correspond to real situations of abnormal electroencephalograms with oscillations of large amplitude in the number of firing neurons as those found in epileptic seizures. We successfully obtained hypersynchronous oscillations in the mesoscopic scale which can be compared with the real EEG of epileptic patients during the ictal state. Moreover, we found that the complexity of the network studied in this work, $N=10^{6}$ and $k=300$, appearst to be a minimum condition to the emergence of selfsustained oscillatory patterns. This level of complexity is found in insects and, from the point of view of evolutionary biology, this could explain the success and complex repertoire of behaviour of these animals.

To make the model more realistic, in our simulations, we used neurons that were excitatory as well as inhibitory with a probability of excitation and inhibition. We have found that self-sustained periodic oscillations of large amplitude are very rare in the brain, in the sense that they require very specific set of parameters. The control of these parameters could help in the prevention of these patterns of abnormal excitatory activity. Comparison between real EEG and the modelled signals could allow for the use of the excitation and inhibition parameters, $\alpha$ and $\gamma$, to classify the pre-ictal, ictal and interictal stages [54]. The effect of antiepileptic drugs could also be modelled in terms of a small change in these parameters and this tool could be useful in the gauging of the drug's effectiveness.

The electrical activity of the brain is recorded in the electroencephalograms by means of electrodes attached to the scalp. This is a non-invasive technique with a long tradition in neurology and psychiatry as a method to detect abnormal behaviour of the brain and also as a diagnostic tool [51]. EEG have also been used in many mathematical models and studies as a primordial raw fact in neuroscience. In particular, we have discussed it in several papers in the context of cellular automata models [39, 52, 53]. Also, recently, both healthy and epilepticform brain activity have been simulated using cellular automata with long-range interactions [55]. Other mathematical and computational approaches have also been considered and the understanding and modelling of the mechanisms of epilepsy is an active research field nowadays [56].

Our approach by means of a stochastic cellular automata random network has been shown to exhibit also low-amplitude noisy patterns and oscillatory behaviour with high amplitude. The last one can be put in correspondence with the epileptipform activity in real brains. Further research could be 
done in the statistical properties of the signals predicted by the model to disclose its analogy with the ictal and pre-ictal stages in epilectic seizures. From the methodological point of view, our model can be also generalized by replacing the cellular automata with more sophisticated neuron models such as Hogdking-Huxley [32] or FitzHugh-Nagumo [33]. The emergence of large-scale oscillations could also be tested in these models. We are currently working along these lines.

\section{References}

[1] S. Ramón y Cajal, Ramón y Cajal's Croonian Lecture: La fine structure des centres nerveux, Proceedings of the Royal Society of London 53 (1894) 444-468.

[2] M. Abeles, Corticonics: Neural circuits of the cerebral cortex, Cambridge University Press, Cambridge, 1991.

[3] V. Braitenberg and A. Schüz, Statistics and Geometry of Neuronal Connectivity, Springer-Verlag, Berlin, 1998.

[4] S. Wolfram, A new kind of science, Wolfram media Inc., 2002.

[5] S. Wolfram, Cellular automata and complexity: Collected papers, Addison-Wesley Pub. Co., Reading, Massachusetts, 1994.

[6] E. R. Kandel, J. H. Schwartz, T. M. Jessel, Principles of Neural Science, Fourth Edition, McGraw-Hill, New York, 2000.

[7] W. Gerstner, W. M. Kistler, Spiking neuron models: Single neurons, populations and plasticity, Cambridge University Press, 2002. Available online at: http://icwww.epfl.ch/gerstner/BUCH.html

[8] A. Hazra, F. Gu, A. Aulakh, C. Berridge, J. L. Eriksen and J. Žiburkus, Inhibitory neuron and hippocampal circuit disfunction in an aged mouse model of Alzheimer's disease, PLoS ONE:e64318, DOI: 10.1371/journal.pone.0064318

[9] R. S. Sloviter, Decreased hippocampal inhibition and a selective loss of interneurons in experimental epilepsy, Science 235(4784) (1987) 73-76. DOI: $10.1126 /$ science. 2879352 
[10] A. M. Wójtowicz, A. Dvorzhak, M. Semtner and R. Grantyn, Reduced tonic inhibition in striatal output neurons from Huntington mice due to loss of astrocytic GABA release through GAT-3, Front Neural Circuits Nov 26 (2013) 7:188. DOI: 10.3389/fncir.2013.00188. eCollection 2013.

[11] H. D. I. Abarbanel, M. I. Rabinovich, A. Selverston, M. V. Bazhenov, R. Huerta, M. M. Sushchik and L. L. Rubchinskii, Synchronisation in neural networks, Physics-Uspekhi 39 (4) (1996) 337-362.

[12] X. Jiao and R. Wang, Synchronous firing patterns of neuronal population with excitatory and inhibitory connections, Int. J. of Non-Linear Mechanics 45 (2010) 647-651.

[13] C. J. Cheng, T. L. Liao, J. J. Yan and C. C. Hwang, Synchronization of neural networks by decentralized feedback control, Phys. Lett. A 338 (2005) 28-35.

[14] W. Lu and T. Chen, Synchronization of coupled connected neural networks with delays, IEEE Trans. on Circuits and Systems-I: Regular Papers 51 (12) (2004) 2491-2503.

[15] X. Li and M. Bohner, Exponential synchronization of chaotic neural networks with mixed delays and impulsive effects via output coupling with delay feedback, Mathematical and Computer Modelling 52 (2010) 643-653.

[16] E. M. Izhikevich, Simple model of spiking neurons, IEEE Trans. on Neural Networks 14(6) (2003) 1569-72.

[17] R. G. Kavasseri and R. Nagarajan, Synchronization in Electrically Coupled Neural Networks, Complex Systems 16 (2006) 369-380.

[18] R. Segev, Y. Shapira, M. Benveniste and E. Ben-Jacob, Observations and modeling of synchronized bursting in two-dimensional neural networks, Phys. Rev. E 64 (2001) 011920.

[19] H. E. Stanley, L. A. N. Amaral, P. Gopikrishnan, P. Ch. Ivanov, T. H. Keitt and V. Plerou, Scale invariance and universality: organizing principles in complex systems, Physica A 281 (2000) 60-68. 
[20] C. Bernard, R. Cossart, J. C. Hirsch, M. Esclapez and Y. Ben-Ari, What is GABAergic inhibition? How is it modified in epilepsy?, Epilepsia 41 (6) (2000) S90-S95.

[21] B. Bollobás, Random Graphs, 2 ed., Cambridge University Press, Cambridge, 2001.

[22] D. J. Watts, Small Worlds: The dynamics of networks between order and randomness, Princeton University Press, Princeton, 2003.

[23] A. L. Barabási, A Réka, Emergence of scaling in random networks, Science 286(5439) (1999) 509-512.

[24] M. Marín-Padilla, The Human Brain: Prenatal development and structure, Springer-Verlag, Berlin, 2011.

[25] L. Acedo, J. A. Moraño, Brain oscillations in a random neural network, Mathematical and Computer Modelling 57(7-8) (2013) 1768-72.

[26] BOINC main page: http://boinc.berkeley.edu

[27] Neuroinformatics: An overview of the Human Brain Project, S. H. Koslow and M. F. Huerta, Eds., Lawrence Erlbaum Associates, Inc., Publishers, New Jersey, 1997. Project's webpage: https://www.humanbrainproject.eu/

[28] R. Menzel and M. Giurfa, Cognitive architecture of a mini-brain: the honeybee, Trends in Cognitive Sciences 5(2) (2001) 62-71.

[29] Wikipedia, List of animals by numbers of neurons, http://en.wikipedia.org/wiki/List_of_animals_by_number_of_neurons (accessed: February 4, 2015).

[30] A. P. Alivisatos, M. Chun, G. M. Church, R. J. Greenspan, M. L. Roukes and R. Yuste, The Brain Activity Map Project and the Challenge of Functional Connectomics, Neuron 74 (2012) 970-974.

[31] M. Wehr and G. Laurent, Odour encoding by temporal sequences of firing in oscillating neural assemblies, Nature 384 (6605) (1996) 162-166. 
[32] A. L. Hodgkin, A. F. Huxley, A quantitative description of membrane current and its application to conduction and excitation in nerve, The Journal of physiology 117 (4) (1952) 500-544.

[33] R. FitzHugh, Mathematical models of excitation and propagation in nerve. Chapter 1, pp. 1-85 in H.P. Schwan, ed. Biological Engineering, McGraw-Hill Book Co., N.Y., 1969.

[34] A. Amarasingham, T. L. Chen, S. Geman, M. T. Harrison and D. L. Sheinberg, Spike count reliability and the Poisson hypothesis, The Journal of Neuroscience 26(3) (2006) 801-809.

[35] S. Lamb, J. J. Webster, Language and reality: Selected writings of Sydney Lamb, Bloomsbury Academic, London, 2006.

[36] P. Maquet, D. Dive, E. Salmon, B. Sadzot, G. Franco, R. Poirrier and G. Franck, Cerebal glucose utilization during sleep-wake cycle in man determined by positron emission tomography and [18F]-2-fluoro-2 deoxyD-glucose method, Brain Research 513 (1990) 136-143.

[37] V. V. Vyazovskiy, U. Olcese, Y. M. Lazimy, U. Faraguna, S. K. Esser, J.C. Williams, C. Cirelli and G. Tononi, Cortical firing and sleep homeostasis, Neuron 63(6) (2009) 865-878.

[38] A. D. Grosmark, K. Mizuseki, E. Pastalkova, K.. Diva and G. Buzsáki, REM Sleep Reorganizes Hippocampal Excitability, Neuron 75(6) (2012) 1001-1007.

[39] L. Acedo, A second-order phase transition in the complete graph stochastic epidemic model, Physica A 370 (2006) 613-624.

[40] V. Priesemann, M. Valderrama, M. Wibral and M. Le Van Quyen, Neuronal avalanches differ from wakefulness to deep sleep - Evidence from intracanial depth recordings in humans, PLOS Computational Biology $9(3)$ (2013) e1002985.

[41] Y. Bar-Yam, Dynamics of Complex Systems, Available on-line at http://necsi.edu/publications/des/.

[42] Falua webpage: http://falua.cesfelipesegundo.com/Neurona/ 
[43] J. Villanueva-Oller, L. Acedo, J. A. Moraño, A. Sánchez-Sánchez, Epidemic random network simulation in a distributed computing environment, Abstract \& Applied Analysis, 2013, ID462801.

[44] L. Acedo, J. Villanueva-Oller, J. A. Moraño, R. J. Villanueva, The Neurona @Home Project: Simulating a Large-Scale Cellular Automata Brain in a Distributed Computing Environment, AIP Conference Proceedings 1510 (2012) 267-270. DOI: 10.1063/1.4776528

[45] M. Galarreta and S. Hestrin, Frequency-dependent synaptic depression and the balance of excitation and inhibition in the neocortex, Nature Neuroscience 1 (1998) 587-594. DOI:10.1038/2822

[46] X. Li, J. Wang, B. Huang, S. Lu, The DCT-based oscillation detection method for a single time series, Journal of Process Control 20 (2010) 609-617.

[47] P. Bak, C. Tang, K. Wiesenfeld, Self-organized criticality: An explanation of the $1 / f$ noise, Phys. Rev. Lett. 59 (1987) 381-384.

[48] C. Bédard, H. Kröger, A. Destexhe, Does the $1 / f$ frequency scaling of brain signals reflect self-organized critical states ?, Phys. Rev. Lett. 97 (2006) 118102.

[49] M.I. Hoffmann, A cellular automaton model based on cortical physiology, Complex Systems 1 (1987) 187-202.

[50] M. Tatsuno, Y. Nagai, W. Aizawa, Rule-dynamical approach to hippocampal network, Neurocomputing 38-40 (2001) 965-971.

[51] S. Tong, N. V .Thakor (Eds.), Quantitative EEG analysis: Methods and clinical applications, Artech House, Norwood, MA, 2009.

[52] L. Acedo, A cellular automaton model for collective neural dynamics, Mathematical and Computer Modelling 50 (2009) 717-725.

[53] L. Acedo, D. F. Aranda, Self-correlations of electroencephalograms, Complex Systems 20 (2012) 289-303.

[54] J. Engel Jr., Seizures and Epilepsy, F. A. Davis Company, Philadelphia, 1989. 
[55] V. Tsoutsouras, G. Ch. Sirakoulis, G. P. Pavlos, A. C. Iliopoulos, Simulation of healthy and epileptiform brain activity using cellular automata, International Journal of Bifurcation and Chaos, 22 (2012) 1250229. DOI:10.1142/S021812741250229X

[56] R. A. Stefanescu, R. G. Shivakeshavan and S. S. Talathi, Computational models of epilepsy, Seizure 21 (2012) 748-759. DOI:10.1016/j.seizure.2012.08.012 